\title{
LA FRONTERA DE LA CULTURA: UN ESTUDIO DE LA FRONTERA DESDE LA PERSPECTIVA ORGANIZACIONAL
}

\author{
Por \\ Mariano Ortega*
}

\section{RESUMEN}

La cultura fronteriza es analizada desde el punto de vista de la administración organizacional a partir de los conceptos de cultura, subcultura y frontera. Se concluye que toda la frontera tiene propiedades específicas emanadas de su condición y naturaleza limítrofe, aunadas a, e independientes de, las entidades divididas por estas fronteras. Se sugiere el estudio de las fronter as como un camino para aumentar y mejorar el conocimiento de las mismas entidades; al mismo tiempo, se reconoce el valor crucial de estos conocimientoss en un mundo cada vez más interdependiente.

\begin{abstract}
Border culture is explored from the perspective of organizational administration using the concepts culture, subculture and border. It is concluded that every border has specific characteristics drawn from its own peculiar condition and nature, in addition to and independently of those possessed by the entities divided by these borders. The study of borders is suggested as a method of improving and increasing our knowledge of the entities themselves. At the same time, this study recognizes the critical importance of the obtained information in an increasingly interdependent world.
\end{abstract}

\section{INTRODUCCION}

Este trabajo se propone explorar el concepto de frontera -organizacional, regional, nacional, etc.- desde la perspectiva de algunas teorías y modelos desarrollados para el estudio de las organizaciones y de su administración.

* El Dr. Ortega es director del Centro de Desarrollo y Estudios Sociales y Administrativos (CEDESA) una institución privada de consultoría, investigación y educación en Querétaro, México. Su doctorado lo obtuvo de la Universidad de Wisconsin. Sus investigaciones abarcan temas de cultura organizacional, administración y proyectos de evaluación e intervención para mejorar el ambiente cultural de las organizaciones.

Dr. Ortega is Director of Center for Management and Social Studies and Development (CEDESA) a private consulting, research and educational institution, in Querétaro, México. His $\mathrm{Ph}$. D. was granted by the University of Wisconsin. Dr. Ortega's research encompasses cultural organizational topics, management and intervention method to assess and improve cultural climate in organizations. 
La exploración gira, esencialmente, en torno a las concepciones organizacionales de cultura, subcultura' y frontera. La premisa fundamental de este trabajo supone que toda frontera tiene características definitorias que le vienen precisamente por su condición limítrofe o su naturaleza de frontera, adicionalmente a aquéllas que puedan ser producto del tipo particular de entidad -departamento, organización, estado, nación, civilización, etc.- a la que se sirva de frontera, e independientemente de éstas.

En este contexto, este trabajo intenta bosquejar algunas de esas características definitorias de la frontera y la manera en que se constituyen en una subcultura distintiva -la cultura de la frontera-. Para ello, utilizando diversas perspectivas organizacionales, se establecen y definen una serie de conceptos fundamentales, como cultura, subcultura y frontera, a partir de los cuales se ofrece una serie de proposiciones lógicas que delinean la frontera de la cultura y establecen algunas de las características de dicha subcultura.

En suma, este trabajo se propone atender la frontera 1) con un enfoque generalmente poco utilizado para este tipo de estudios generales: el de las teorías de la organización y la administración; 2) a partir de una premisa también poco frecuente: la de que las fronteras departamentales, organizacionales, regionales, nacionales, etc. tienen un denominador común esencial por el hecho mismo de ser fronteras, independientemente del tipo o nivel de entidad de que se trate (departamento, nación, etc.) y, en cierta medida, de los valores, comportamientos, etc., que caractericen y definan la cultura particular de la entidad de que se trate; y 3) para ofrecer como producto una serie de proposiciones desarrolladas, no empírica, sino lógicamente. Estas tres características constituyen, a un tiempo, las aportaciones potenciales y las limitaciones más evidentes de este trabajo.

Aunque está de manifiesto que en el carácter fundamentalmente propositivo de este trabajo se puede encontrar una de sus más grandes limitaciones, también es cierto que ese mismo carácter puede permitirle una flexibilidad conceptual que, potencialmente, permita enriquecer los marcos de referencia con los que tradicionalmente se contemplan las fronteras.

De la misma manera, el enfoque de la frontera como provincia limítrofe de una cultura o subcultura -departamento, área, organización, región, nación, etc.- y su consecuente liberación de su asociación indispensable con lo nacional y lo geográfico, ofrece la oportunidad de contemplar la frontera en un contexto conceptual más amplio y potencialmente más puro, aunque se corra el riesgo palpable de oscurecer diferencias o diluir particularidades o variables que puedan ser altamente relevantes para la comprensión de ciertos tipos de fronteras.

\footnotetext{
${ }^{1}$ En este trabajo la palabra subcultura denota un témmino comúnmente utilizado en la teoría organizacional. No intenta de ninguna manera una disminución categórica en lo social.
} 
De ser este el caso, el reconocimiento eventual de variables adicionales que respondan a diferencias críticas entre fronteras podría reflejarse en proposiciones adicionales que precisen las que aquí se ofrecen. De ser así, los mapas conceptuales de las fronteras se habrán enriquecido. En todo caso, es importante explorar lo que el estudio de algunas fronteras (organizacionales, departamentales, etc.) puede contribuir al conocimiento de las otras (nacionales, regionales, etc.) sin tener que redescubrir las mismas cosas para cada tipo de frontera.

\section{CONCEPTOS FUNDAMENTALES}

Existen tres conceptos fundamentales para los propósitos de este trabajo: cultura, subcultura y frontera. Las definiciones operativas que aquí se utilizan, como se ha dicho, son las que han sido desarrolladas en el estudio de las organizaciones formales y de su administración. Por ello, sus antecedentes inmediatos se encuentran en las teorías organizacionales y de la administración y no en la antropología o en la sociología general, como suele ser el caso, aunque, en muchas ocasiones, de las definiciones propias de estas disciplinas se hayan desprendido las definiciones organizacionales utilizadas. Esto es cierto aún para los casos (por ejemplo, Hofstede, 1976, 1980 y 1984) en los que se han enfocado culturas nacionales, puesto que esa atención se ha concentrado precisamente en los valores hacia la organización y hacia el trabajo.

El término cultura se utiliza para visualizar e identificar una entidad relativamente unitaria que, según el caso, puede ser un departamento, una organzación. una región, una nación, etc., en la que existe una cierta comunidad de valores, premisas, creencias, etc. (figura 1). Quienes comparten esos valores y premisas comparten también visiones del mundo, de la realidad, de la organización, del trabajo, etc. y se comportan -inconsciente y automáticamente-en forma semejante, ante una misma situación, contexto, problema, etc.

El término subcultura se utiliza para visualizar e identificar, a su vez, subunidades constitutivas de una cultura, que aunque comparten sus valores, premisas y comportamientos básicos, exhiben, también, rasgos propios que permiten reconocerlas y diferenciarlas como modalidades o variantes dentro de la unidad, como culturas particulares (o especializadas) dentro de una cultura más amplia (figura 1). Quienes comparten esa subcultura exhiben, en sus valores, premisas y comportamientos, modalidades plenamente identificables y reconocibles para los demás miembros de la cultura más amplia de la que forman parte, aunque difícilmente detectables -en sus diferencias o modalidades-para miembros de otras culturas.

Finalmente, el término frontera se utiliza para designar a aquellas provincias o partes de una entidad relativamente unitaria (es decir, de una cultura) en contacto permanente con otra u otras entidades también relativamente unitarias (es decir, 


\section{Mariano Ortega}

con otra u otras culturas. (figura 1). Una frontera puede adicionalmente estar definida por variables espaciales o geograficas, temporales, linguísticas, ideologicas, etc. Quienes habitan una frontera suelen desarrollar -en comparación con otros miembros de esa misma cultura- una mayor conciencia de, por lo menos, algunos de los rasgos distintivos de su propia cultura. Esta mayor conciencia es un producto de su situación de frontera, es decir, del enfrentamiento, literalmente hablando, a una cultura diferente, lo que obliga al reconocimiento no sólo de ésta, sino, en cierta medida, de la propia.

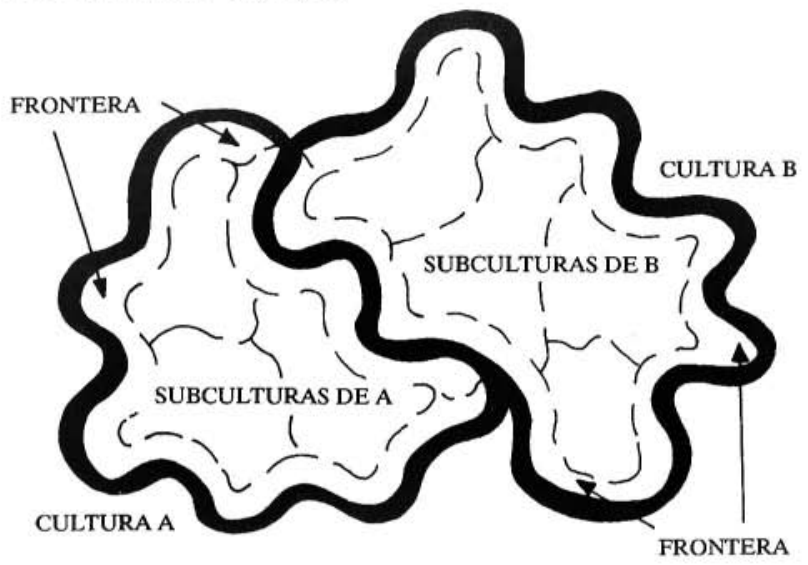

FIGURA 1. Cultura, subcultura y frontera.

\section{CULTURA}

Una cultura se contempla como la entidad básica de estudio para este trabajo. En este sentido, los parámetros que definen el objeto de estudio dejan de estar centrados o limitados exclusivamente en lo meramente físico, geográfico, político, legal, etc., aun y cuando este tipo de variables puedan incluirse en su definición. Así considerada, la entidad básica de estudio puede ser igualmente una nación que un departamento, una región o un estado que una organización.

Para Hofstede (1984), que enfoca el término desde la perspectiva amplia de nación o de conjunto de naciones, cultura es:

"La programación colectiva de la mente que distingue a los miembros de un grupo humano de otros grupos humanos... agregado interactivo de características comunes que afectan las respuestas de un grupo humano a su medio ambiente. La cultura determina la identidad de un grupo humano de la misma manera que la personalidad determina la identidad de un individuo" (pág.21). 


\section{LA FRONTERA DE LA CULTURA}

Hofstede (1980 y 1984) establece cuatro índices para tipificar una cultura: 1) distancia del poder, 2) rechazo a la incertidumbre, 3)masculinidad/feminidad y 4) individualismo/colectivismo. La cuantificación de estos índices para aproximadamente 40 países, parecería confirmar -en alguna, aunque a veces discutible, medida- la existencia de ciertas culturas extranacionales (frecuentemente supuestas por el sentido común, como podrían serlo la escandinava, la iberoamericana, la anglosajona, etc.).

Por su parte, para Edgar Schein (1984), quien enfoca el mismo término desde la perspectiva mínima de los pequeños grupos, cultura es:

"Un patrón de supuestos y creencias básicas inventados, descubiertos o desarrollados por un grupo dado en su aprendizaje para enfrentar sus problemas de adaptación externa e integración interna, que ha respondido lo suficientemente bien como para ser considerado como válido $\mathrm{y}$, por lo tanto, que debe ser enseñado a los nuevos miembros como la forma correcta de percibir, pensar y sentir en relación a esos problemas" (pág. 3).

A diferencia de Hofstede, Schein no tipifica las culturas, sino que busca describirlas atendiendo a los supuestos y premisas básicos referentes a: 1) la relación con el medio ambiente; 2) la naturaleza de la realidad; 3) la naturaleza humana; 4) la naturaleza de la actividad humana; y 5) la naturaleza de las relaciones humanas características de cada cultura (Schein, 1984 y 1985).

Finalmente, una cultura puede contemplarse como:

La manera particular y diferenciada de un conjunto de personas para ver, comprender y actuar, tanto en relación con el mundo en el que individual y colectivamente están inmersas, como en relación con sí mismas, en tanto individuos y en tanto colectividad. Esta manera particular y diferenciada implica: a) una serie de valores, premisas y supuestos compartidos que sustentan acciones y comportamientos, o b) fundamentan posturas, filosofías y manifiestos; c) un grado de congruencia entre los que sustentan aquéllos y los que fundamentan éstos; d) un grado de consistencia interna en unos y en otros; y e) un grado de apertura frente a valores, premisas, comportamientos y posturas diferentes o heterodoxos (Ortega, 1988:5).

Las culturas pueden tipificarse según un perfil dimensional, característico y particular a cada cultura, integrado por las dimensiones: 1) racional, 2)relacional, 3) estructural, 4) política y 5) simbólica (Ortega, 1982a).

Este perfil dimensional es resultante del tipo de valores, premisas y supuestos compartidos que sustentan las acciones y comportamientos de esa cultura y fundamentan sus posturas, filosofías y manifiestos. En ese perfil se reflejan, 
asimismo, el grado de congruencia entre ambos conjuntos, su consistencia interna y su grado de tolerancia o apertura frente a valores, premisas y comportamientos diferentes o heterodoxos en áreas marginales o no fundamentales para esa cultura.

\section{SUBCULTURA}

Una subcultura se concibe como una entidad reconocible dentro de una entidad más amplia con la que se comparten los valores, premisas, comportamientos, etc. fundamentales y definitorios, pero en torno a los cuales se han ido generando valores, premisas, comportamientos, etc. adicionales y diferentes que, por una parte, la dotan de los rasgos que precisamente le crean una entidad propia y la hacen reconocible y, por la otra, se integran lo suficientemente bien con los valores, premisas y comportamientos básicos o fundamentales de la cultura más amplia como para ser aceptados por éstos.

Mientras que los valores, premisas, comportamientos, etc. de la cultura más amplia son compartidos por todos; aquellos valores, comportamientos, premisas, etc. que dotan a una subcultura de su identidad particular dentro del todo, sólo son compartidos por los miembros de dicha subcultura. "Las subculturas pueden generar premisas y creencias y determinar comportamientos de una manera muy semejante a la que pueden hacerlo las culturas" (Deal y Kennedy, 1982: 151).

Aunque hay autores como Hofstede (1984: 21) que "reservan el término cultura para las sociedades (o naciones) [y] para todo lo demás utiliz[an] "subcultura"; los términos suelen utilizarse de manera semejante a los de "sistema" y "subsistema", reservando "cultura" para referirse a la entidad objeto de estudio y "subcultura", para sus diversas subunidades constitutivas; entendiendo siempre que toda "cultura" es, en el fondo, una "subcultura" de una cultura mayor; y que toda "subcultura" puede siempre contemplarse como una "cultura" en sí misma, constituida a su vez por una serie de "subculturas".

En cierta medida, una subcultura puede ser considerada como el punto de encuentro de conjuntos de valores, premisas, comportamientos, etc., diversos, y en los que cada uno de esos conjuntos, conforma potencialmente los valores, comportamientos, premisas, etc., básicos y definitorios de culturas distintas. Sin embargo, y aunque un observador externo pueda reconocer individualmente la naturaleza y el origen de cada conjunto, debe ser evidente que para quienes los comparten -es decir, para los miembros de esa subcultura específica- todos juntos constituyen ese "patrón de supuestos y creencias básicos" que ha sido indispensable conformar, para responder adecuadamente a "sus problemas de adaptación externa e integración interna" (Schein, 1984; 3 y 1985; 9), su cultura particular.

Una subcultura es, en suma, una subunidad de cultura más amplia, que tiene que enfrentar problemas particulares y adicionales de adaptación externa e/o 
integración interna, sea por la naturaleza específica de la parte del medio ambiente a la que concretamente tuvo que adaptarse; sea por las tareas específicas que lo hayan sido asignadas; sea por las exigencias adicionales de la integración internu que su situación, su tarea o su naturaleza conllevan.

Precisamente, es el contacto e intercambio constantes y permanentes con una cultura diferente lo que genera en la subunidad limítrofe de una cultura esu subcultura particular, la subcultura o la cultura de la frontera.

\section{FRONTERA}

Una frontera se concibe, así, como la provincia limítrofe de una cultura; como aquello que marca la diferencia entre una cultura y su medio ambiente -a su vez. constituido por otras u otras culturas-:

"La frontera incluye, aunque sin limitarse a ellas, barreras físicas entre actividades internas y fuerzas externas [e] implica diferencias en lenguaje, normas e intensidad de interacción... Aunque el concepto alusivo de frontera organizacional [regional, departamental, nacional, etc., cultural, en suma] es difícil de aprehender en una definición nítida, la presencia de esa frontera se manifiesta por el incremento en el esfuerzo necesario para mantener un flujo de movimiento, comunicación o actividad a través de ella (Organ y Hammer, 1982: 456).

El problema clave al que se enfrentan las fronteras es de adaptabilidad y ajuste (Thompson, 1967) a las variables adicionales creadas por la presencia de la otra cultura, por el trato con su contrapartida fronteriza.

Aún más, hay grados de "fronteridad" -la frontera no es todo o nada- (Organ y Hammer, 1982: 456). Estos grados de "fronteridad", sin embargo, parecerían estar determinados no por el carácter propio de toda frontera, sino por el grado de afinidad entre los valores y premisas particulares de las culturas en juego. Dependiendo de ese grado de afinidad, las fronteras podrán ser más o menos permeables, más o menos porosas, aunque debe ser evidente que no existen las fronteras totalmente permeables o abiertas, ni totalmente impermeables o cerradas (Ortega, 1982b), sino que las culturas se sitúan en algún lugar de un contínuo permeable-impermeable o abierto-cerrado.

"[La localización de una cultura en ese continuo, sin embargo] no es, de ninguna manera, permanentemente fija, sino que, dependiendo de las condiciones del medio ambiente, de la incertidumbre que éste [le] genere... y de sus propias capacidades para procesar esa incertidumbre, [esa cultura] podrá abrirse, cerrarse o mantener su posición en el continuo abiertocerrado". (Ortega, 1985: 21). 
En otras palabras: 1) las fronteras exhiben diversos grados de porosidad o permeabilidad -de "fronteridad", separación o diferenciación-: y 2) ese grado de porosidad o permeabilided es variable y depende de las necesidades o condiciones de la propia cultura en un momento dado. Así, una frontera podrá modificar su porosidad o permeabilidad, facilitando o dificultando ese flujo de actividad o comunicación a través de ella o exigiendo un mayor esfuerzo para mantenerlo.

A título de ejemplo, por lo que se refiere al grado de "fronteridad" (punto 1 del párrafo anterior), compárense las fronteras entre México/EUA, Canadá/EUA y EUA/URSS; entre General Motors (GM) y alguna de sus subsidiarias; GM y una organización que produzca lo mismo, pero que no sea su subsidiaria; y GM y una organización con iguales productos, pero subsidiaria de Ford; etc. El ejemplo puede extenderse para referirse a su naturaleza dinámica o cambiante (punto 2 del párrafo anterior), comparando en este caso la porosidad o permeabilidad de la frontera México/EUA 1989 versus 1909; la de EUA/URSS 1989 versus 1959; o la de General Motors y alguna de sus subsidiarias en 1989 versus 1969 . Es evidente que a estos ejemplos en los que se utilizan naciones y organizaciones para ilustrar los comportamientos fronterizos entre culturas, pueden sumarse otros en los que se utilicen departamentos, regiones, etc.

En cierto sentido, "las fronter as deben, idealmente, mantener el equilibrio entre la permeabilidad a insumos apropiados y la impenetrabilidad a insumos irrelevantes o perturbadores" (Organ y Hammer, 1982: 457) y el mantenimiento de ese equilibrio puede requerir grados diversos de permeabilidad e impenetrabilidad en distintos momentos.

Por otra parte, cada cultura busca "incorporar dentro de sus fronteras aquellas actividades que [la propia cultura considera que], de quedar fuera, constituirían contingencias cruciales" (Thompson, 1967: 39). Dependiendo tanto de la naturaleza de la cultura considerada (nación, región, organización, etc.), como de sus valores, premisas, etc., específicos, esta incorporación de contingencias cruciales o estratégicas afecta, también, el grado de "fronteridad" de que se ha hablado. Puede desplazar o extender la frontera desigualmente, más para unas actividades que para otras (el combate de drogas en el lugar en donde se cultiva versus inmigración en los puertos de entrada, para una nación; la creación o el mantenimiento de una imagen corporativa versus compras, para una organización); o puede filtrar desigualmente sus insumos o productos (prohibiciones comerciales para ciertos países pero no para otros, en una nación; rechazo de candidatos egresados de ciertas universidades pero no de otras, para una organización).

Sin embargo, una cultura "con poder sobre otra que controla o domina esa contingencia crucial o estratégica, no necesita [extender su frontera e] incorporarla formalmente" (Thompson, 1967: 48) basta su poder relativo sobre la segunda cultura para obtener los mismos resultados (por ejemplo, la nación A puede 
considerar las defensas de la nación B como sus propias defensas, con lo cual no se ve en la necesidad de extender sus fronteras e instalar las propias; unu organización que contrata a una agencia de personal para seleccionar a sus empleados no tiene que establecer otra unidad de selección dentro de sus propias fronteras).

Por su carácter limítrofe, la frontera parece ejercer dos funciones especialmente: importantes, tanto para su propia cultura como para la ajena: 1) intérprete y 2) dosificador de insumos y productos.

En su función de intérprete o traductor, la frontera vuelve inteligibles los valores, premisas, comportamientos, etc., de ambas culturas y las comunicaciones en ambas direcciones.

En su función de dosificador, la frontera: a) media y amortigua las intenciones de ambas culturas; b) equilibra o regula el flujo de insumos o productos; $c$ ) rechaza insumos no deseados o los habilita, transforma o socializa para hacerlos aceptables; y d) protege la identidad e integridad de su propia cultura (veáse, por ejemplo, Organ y Hammer, 1982; Thompson, 1967).

Mientras que estas funciones características diferencian e identifican a la frontera de las demás subunidades o subculturas dentro de su propia cultura; los valores, premisas, comportamientos, etc., de esta cultura -compartidos por su frontera-, son, precisamente, los que la identifican y diferencian de otras fronteras.

En suma, la subcultura particular de una frontera es el producto de la confluencia de dos conjuntos de comportamientos, valores, premisas, etc., diversos: 1) el que constituye y define su propia cultura y 2) el generado por su carácter limítrofe. En éste, se integran los rasgos generales comunes a toda frontera y los rasgos particulares producto del grado de afinidad entre las culturas propia y contigua.

\section{LA SUBCULTURA DE LA FRONTERA}

La integración de lo discutido hasta ahora en torno a los conceptos de cultura, subcultura y frontera -desarrollados para el estudio de las organizaciones y de su administración- permite ofrecer las siguientes proposiciones generales:

1. La frontera de una cultura constituye una subcultura específica.

2. Las características definitorias de esta subcultura específica reflejan, por una parte, aspectos fundamentales de la cultura más amplia de la que forma parte; $y$. por la otra, exhiben rasgos particulares debidos a su carácter limítrofe, a su condición de frontera.

3. Estos rasgos particulares son, por ello, compartidos en una gran medida por toda la frontera de una cultura, es decir, por toda subcultura de frontera, incluyendo 
a aquélla de la cultura contigua, frente a la que se constituye precisamente como frontera.

4. En ese sentido, la frontera de una cultura, a un tiempo, une y separa. Exhibe, por una parte, características comunes a las de la frontera de la cultura contigua, y por la otra, ostenta los rasgos diferenciales más básicos de la propia cultura; se constituye así, no sólo en la última provincia de su propia cultura, sino, en cierta medida, en la primera provincia de la cultura contigua.

5. El conjunto de valores, premisas, comportamientos, etc., producto de los problemas de adaptación externa e integración interna generados por su carácter limítrofe, -por su naturaleza de frontera- y las exigencias o funciones específicas correspondientes a este carácter, a esta naturaleza, son precisamente lo que une a una frontera con su frontera contigua.

6. El conjunto de valores, premisas, comportamientos, etc., que identifican y constituyen su propia cultura, particulares a ésta, son precisamente lo que separa a una frontera de su frontera contigua. Esta separación o diferenciación es inversamente proporcional al grado de afinidad que exista entre esos valores, premisas, comportamientos, etc. y los que caractericen a la cultura contigua.

7. Este grado de afinidad puede determinarse comparando el perfil dimensional de cada cultura (Ortega, 1982), sus índices (Hofstede, 1980 y 1984) o la descripción de sus premisas, supuestos y valores básicos subyacentes (Schein, 1984 y 1985).

8. Este grado de afinidad, que puede ser diferente para cada par de culturas en contacto, puede generar tantas subculturas de frontera, dentro de una misma cultura, como fronteras tenga ésta con otras culturas diversas.

9. Dos culturas pueden coincidir o diferir en: a) los valores, supuestos y premisas que sustentan sus comportamientos; b) los valores, supuestos y premisas que fundamentan sus posturas, filosofías y manifiestos; c) los grados de congruencia entre uno y otros; d) los grados de consistencia interna en cada uno de ellos; y e) los grados de apertura frente a valores, premisas, comportamientos y posturas diferentes y heterodoxos (Ortega, 1988).

10. Aunque todos influyen, quizás los dos más importantes para determinar el grado de afinidad, o su inverso, el grado de "fronteridad" entre dos culturas, sean la coincidencia en: a) los valores, supuestos y premisas que sustentan sus comportamientos -es decir, la vida cotidiana de la acción y los hechos-; o en b) los grados de apertura frente a valores, premisas, comportamientos y posturas diferentes y heterodoxos. En el primer caso, es evidente que se trata de una comunidad de valores; en el segundo, es un respeto a valores diferentes a los propios. 
11. El grado en que una frontera une o separa es variable y dinámico; depende de las necesidades y condiciones imperantes en un momento dado; y se refleja en el esfuerzo requerido para mantener un determinado flujo de movimiento, comunicación o actividad a través de ella.

12. Esta dinámica dual por la que une y separa, permite que la frontera de una cultura pueda comprenderse como intérprete, tanto de la propia cultura ante la ajena, como de la ajena ante la propia.

13. Paradojica pero comprensiblemente, esa misma función la pone en contraposición con ambas culturas, puesto que independientemente de la cultura de que se trate, la función de la frontera será la de interpretar la cultura contraria.

14. Esta contraposición automática y permanentemente presente en su relación con ambas culturas, es precisamente uno de los rasgos definitorios de la frontera. Por una parte, la diferencia y distinción dentro de su misma cultura le confiere una identidad propia, de subcultura -la subcultura de la frontera-; por otra parte, la diferencia y distinción de la cultura contigua, pero ajena, desalienta o impide su fusión con la frontera de ésta, a pesar de compartir con ella algunos de sus rasgos.

15. El carácter de subcultura de una frontera es más fácilmente reconocible o evidente para los miembros de su propia cultura (pero no para quienes comparten esa subcultura) que para los miembros de la cultura contigua; para éstos, en cambio, serámás evidente su carácter de cultura-de provincia-indiferenciada de esacultura más amplia a la que pertence.

16. Por los rasgos que comparten, es decir, por su carácter limítrofe, las subculturas de frontera de culturas contiguas pueden ser percibidas (por los miembros de sus respectivas culturas) e, incluso, comportarse -bajo ciertas condiciones, en algunas situaciones o para determinados propositos- como una cultura diferente de las dos culturas originales, como una tercera cultura, la cultura de la frontera. Aun en este caso, ninguna de las dos subculturas de frontera pierde su carácter de subcultura, debido, en esa ocasión, no a su naturaleza de frontera, sino a los rasgos diferenciales propios de su cultura base, de la cultura original de la que cada una es frontera.

\section{CONCLUSIONES}

Más que una serie de conclusiones, estos comentarios aspirarían a constituir una contribución a un punto de partida. Un punto de partida para el estudio de la frontera, en todas y cada una de sus formas, con el objeto de enriquecer el conocimiento no sólo de las entidades a las que delimita, o de algunas fronteras específicas, sino de la naturaleza de toda frontera, de la "fronteridad" misma. 
A ello responde, precisamente, la elección -en este trabajo- de la cultura/ subcultura como la entidad básica para el estudio de la frontera. La cultura/ subcultura objeto de estudio puede, con todorigor y según el caso, entenderse como una civilización (la occidental, por ejemplo); un conjunto de naciones (Iberoamérica, Escandinavia); una nación (México, Filipinas); una región (Nueva Inglaterra, el Istmo); un estado o una provincia (Quebec, Pichincha); una organización multinacional (IBM, UPI, GM); una corporación nacional (Telmex, Vitro, A \& P) o un partido político (COPEI, PSOE, laborismo); etc., hasta llegar al más pequeño de los grupos o a una familia $\mathrm{y}$, ¿por qué no? $-\mathrm{y}$ con las adecuaciones pertinentes- $\mathrm{a}$ una persona.

Con la vista fija en la frontera, este trabajo ha explorado ese concepto utilizando la perspectiva y algunas de las aportaciones de los estudios organizacionales y de la administración. Sin embargo, las proposiciones ofrecidas aspiran a tener una relevancia general; a iluminar -más allá de la frontera organizacional a partir de la cual se generaron- el concepto mismo de frontera, nacional, regional, estatal, grupal, etc.

No se descarta, como se ha dicho, la posibilidad de incorporar eventualmente variables adicionales que reflejen el tipo de frontera de que se trate. De ser ese el caso, el conocimiento de todas las fronteras se habrá también enriquecido y sus diferenciaciones habrán superado lo anecdótico.

El problema en el estudio de la frontera parecería ser el de enfocar primordial, y casi exclusivamente, a la entidad básica (nación, organización, etc.) como el objeto único de estudio. En estos trabajos, la frontera interesa en cuanto parte del todo, nunca en símisma, por lo que se enfoca y se conceptualiza en función del todo y con referencia a éste, desde los marcos de referencia para él desarrollados y con su terminología correspondiente, exclusiva y excluyente.

Quizás en estos marcos de referencia y en esa terminología -muy clara para quienes comparten esos marcos estrechos, pero muy oscura para quienes no- se encuentran las barreras más fuertes para este tipo de estudio interdisciplinario de la frontera, en tanto frontera.

Hoy por hoy, entre los estudios de la frontera más desarrollados parecerían estar los de las fronteras nacionales, a pesar de que en algunos casos se podría decir que se peca de una excesiva preocupación espacial o geográfica. Los estudios de las fronteras organizacionales, quizás en un segundo lugar de desarrollo sufrieron un retraso por el enfoque de sistema cerrado en el que por tanto tiempo se contempló a la organización. No es sino hasta la concepción sistemática de ésta, el interés en el medio ambiente y el descubrimiento del campo interorganizacional que la frontera cobra un interés especial. En ambos casos, sin embargo, la frontera se vuelve relevante en función de las naciones o de las organizaciones estudiadas, no 


\section{LA FRONTERA DE LA CULTURA}

de la frontera misma; también en ambos casos, todo se codifica en su propia terminología excluyente, con lo que se dificulta determinar siquiera si las aportaciones del estudio de un tipo de frontera son relevantes para el estudio de la otra; si las aportaciones del estudio en ambas fronteras pueden contribuir -y en qué medida- al estudio de las otras.

Si es válida la premisa -fundamental a este trabajo- de que todas las fronteras tienen un denominador común por su carácter limítrofe, por su naturaleza de frontera, debe ser evidente que las aportaciones del estudio de un tipo de frontera sí son relevantes para el estudio de las otras; sin embargo, queda por concretar esas aportaciones para cada tipo de frontera, para establecer sus implicaciones específicas, sus datos particulares. De hacerse así, estas aportaciones potenciales al conocimiento de las fronteras pueden también conducir a un mejor o mayor conocimiento de las propias entidades a las que sirven de frontera, a un mejor conocimiento de las naciones, organizaciones, regiones, etc.

Finalmente, debe reconocerse que en un mundo cada vez más interdependiente, el estudio y el conocimiento de la frontera (extranacional, nacional, organizacional, etc.) se vuelve crucial: a mayor interdependencia, mayor el valor estratégico de la frontera, no importa si se trata de una frontera organizacional en las corporaciones globales contemporáneas, o de una frontera nacional en el mundo del GATT, o del Mercado Común Europeo.

\section{BIBLIOGRAFIA}

ARGYRIS, C. Y D. A. Schon 1974. Theory in practice. Increasing Professional Efectiveness. San Francisco: Jossey-Bass.

1978. Organizational learning. A Theory of Action Perspective. Reading, MA.: Addison-Wesley.

DEAL, T.E.y A.A. Kennedy. 1982. CorporateCultures. Reading, MA.: AddisonWesley.

HOFSTEDE, G. 1976. "Nationality and espoused values of managers". Journal of Applied Psychology.

1980 Culture's Consequences. International Differences in Work-related Values. Beverly Hills: Sage.

1984. Culture's Consequences (Abridged edition). Beverly Hills: Sage.

ORGAN, D. W. y W. C. Hammer 1982. Organizational Behavior. Plano, Tx.: Business Publications.

1982b. La organización como sistema. Querétaro: CEDESA.

1985. La evaluación institucional: Un enfoque dimensional del diagnóstico

y la evaluación de las organizaciones. Querétaro: CEDESA.

1988. La cultura organizacional. Querétaro: CEDESA. 
PERROW, C. 1979. Complex organizations (2nd Edition). Glenview, III.: Scott, foresman.

SCHEIN, E. H. 1984. "Coming to a new Awareness of Organizational Culture". Sloan Management Review, 25. 1985. Organizational Culture and Leadership. San Francisco: Jossey-Bass.

THOMPSON, J. D. 1967. Organizations in Action. Nueva York: McGraw-Hill. 